\title{
La SIHFLES en Espagne : historique des recherches et perspectives
}

Juan Francisco García Bascuñana et Javier Suso López

\section{(2) OpenEdition \\ 12 Journals}

Édition électronique

URL : https://journals.openedition.org/dhfles/3703

DOI : $10.4000 /$ dhfles.3703

ISSN : 2221-4038

Éditeur

Société Internationale pour l'Histoire du Français Langue Étrangère ou Seconde

Édition imprimée

Date de publication : 1 janvier 2013

ISSN : 0992-7654

Référence électronique

Juan Francisco García Bascuñana et Javier Suso López, «La SIHFLES en Espagne : historique des recherches et perspectives ", Documents pour l'histoire du français langue étrangère ou seconde [En ligne], 50 | 2013, mis en ligne le 01 janvier 2017, consulté le 26 mars 2023. URL : http:// journals.openedition.org/dhfles/3703; DOI : https://doi.org/10.4000/dhfles.3703

Ce document a été généré automatiquement le 26 mars 2023.

Tous droits réservés 


\title{
La SIHFLES en Espagne : historique des recherches et perspectives ${ }^{1}$
}

\author{
Juan Francisco García Bascuñana et Javier Suso López
}

1 C'est avec Joaquín López Barrera, professeur de français du début du $\mathrm{XX}^{\mathrm{e}}$ siècle, que commence la recherche en histoire de l'enseignement du français en Espagne : dans un bref mémoire de recherche (19 pages), intitulé «Apuntes para una historia de la enseñanza de la lengua francesa en España ", qu'il élabore pour devenir catedrático de français ${ }^{2}$, il se montre pleinement conscient de l'originalité de sa démarche, tout en déplorant le peu d'attention et de prestige qu'il avait à tirer d'un sujet si «pauvre et aride ».

2 Même si López Barrera n'y analyse de façon détaillée que quelques ouvrages ayant servi à l'enseignement du français en Espagne, il faut dire qu'il s'agit d'ouvrages marquants, tels que La gramática [...] de Baltasar de Sotomayor (1565), le Vocabulario de los vocablos [...] de Iacques de Liaño (1565), le Diccionario [...] de Ioan Palet (1604), les Adiciones a la gramática francesa que compuso el R.P. Núñez [...] de Antonio Galmace (1745). Il consigne ensuite une quinzaine de grammaires des $\mathrm{XVII}^{\mathrm{e}}$ et $\mathrm{XVIII}{ }^{\mathrm{e}}$ siècles. Nous allons nous limiter à quelques observations sur ce travail, qui montrent les caractéristiques des études historiques de l'époque et l'approche avec laquelle il envisageait cet objet d'études naissant. Il éprouve ainsi tout d'abord le besoin de dresser un catalogue des ouvrages, et d'y distinguer des phases; il fait état des débats - dont les auteurs se faisaient l'écho - au sujet de la présentation de la matière et dans les procédés de travail ; il croit à une évolution se traduisant par un progrès constant ; il montre un désir d'obtenir des conclusions valables pour les professeurs contemporains. Le travail de López Barrera ne semble intéresser personne et tombe dans l'oubli ; ce n'est que tout récemment (A. Yllera, 1998 ; J. Suso López, 2010) qu'il est « redécouvert ».

Dans la genèse des études historiques sur l'enseignement du français en Espagne, nous devons noter une deuxième étude, toute petite (9 pages). Il s'agit d'un texte d'Angel González Palencia, publié en 1942, ayant pour titre: "Notas sobre la enseñanza del Francés a fines del siglo XVIII y principios del XIX ». Attiré par des recherches historiques sur d'autres sujets, l'auteur, professeur de français, était probablement 
tombé par hasard, dans ses recherches à l'Archivo de la Sala de Alcaldes de Casa y Corte de Madrid, sur des autorisations concédées à la fin du XVIII et début du XIX à une série de professeurs pour donner des cours particuliers de français. Il recueille ainsi les données biographiques de ces professeurs (Ascanio Bono, Juan Bautista Burete, Juan Pedro Tellier, Juan Bautista Tievant, Gaspar Lebrun) et cite également les ouvrages qui ont été autorisés à l'époque pour procéder à cet enseignement : le Dictionnaire de l'abbé Gattel, la Gramática francesa de Constantino Letellier, un Veni mecum de los estudiantes, c'est-à-dire un «libro de la traducción del francés al español y viceversa », de Fray Jerónimo Laverdure.

4 Notre domaine de recherche obtient ses lettres de noblesse avec la thèse de Gonzalo Suárez Gómez: La enseñanza del francés en España. ¿Con qué libros aprendían francés los españoles?, soutenue en 1956, partiellement publiée en 1961 (Revue de Littérature Comparée, XXXV) et récemment rééditée par Juan García Bascuñana et Esther Juan Oliva, avec une étude introductive qui met en relief autant ses grands mérites que certaines lacunes (2008:15-37). Nous renvoyons à cette étude pour une appréciation de l'intérêt de cette thèse, et nous nous limitons à attirer l'attention sur trois faits : Suárez Gómez y dresse un catalogue très abondant des ouvrages ayant servi à l'enseignement du français en Espagne, en y incorporant des œuvres littéraires bilingues (212 entrées, 2008 : 149-194); il y consigne également des données biographiques des auteurs, leurs positionnements et leurs choix au sujet des problèmes soulevés par l'enseignement du français (2008: 42); il y propose un classement des ouvrages en trois groupes (qui constituent autant de types d'outils): A. Des grammaires; B. Des vocabulaires, des diction-naires, des trésors, des nomenclatures bilingues et plurilingues; C. Des ouvrages «littéraires» bilingues (refrains, récits, romans, recueils de poésies ou florilèges, lettres, colloques, anecdotes, œuvres morales et religieuses).

Comme dans le cas de l'étude de López Barrera, la thèse de Suárez Gómez tombe bientôt dans l'oubli ${ }^{3}$ : il faudra attendre la création de la SIHFLES pour qu'avec un groupe de chercheurs espagnols, appartenant à plusieurs universités (Tarragone, Cantabrie, université Autonome de Barcelone, Valence, Valladolid, Grenade, Séville, UNED de Madrid) les recherches historiques sur le FLE en Espagne prennent un nouvel élan ${ }^{4}$. La SIHFLES permet en effet un regroupement des chercheurs, et à partir des contacts lors des colloques, un travail d'équipe naît, axé sur le groupe de recherche GRELINAP/ HIFRES (Groupe de recherche en linguistique appliquée / Histoire du français en Espagne) que dirige Juan García Bascuñana, à l'université Rovira i Virgili de Tarragone. Le groupe a travaillé depuis 1994 sur six projets de recherche financés par les ministères espagnols de l'Éducation et de la Science, de la Science et de l'Innovation, et de l'Économie et de la Compétitivité. Ces projets de recherche ${ }^{5}$, étalés sur près de vingt ans, se sont intéressés à l'histoire de l'enseignement du FLE en Espagne, puis aussi à la présence du français et de la culture française au sud des Pyrénées, le tout abordé de différents points de vue. Les contenus de manuels destinés à cet enseignement ont été étudiés tout d'abord, avec une approche spéciale des sources où leurs auteurs ont puisé et des méthodologies qui s'en dégagent. Ce qui a donné lieu au répertoire publié en 2004 (Repertorio de gramáticas y manuales para la enseñanza del francés en España. 1565-1940) et en 2008 (Repertorio de manuales para la enseñanza del francés en España. Siglo XX). Dans une deuxième phase, l'équipe de recherche s'est intéressée à l'institutionnalisation même de cet enseignement, avec un regard spécial porté aux maîtres et aux professeurs ainsi qu'aux établissements scolaires. D'autre part, les travaux des chercheurs espagnols ont permis de procéder à un déblaie-ment systématique du 
terrain de recherche et d'établir des espaces de connaissance, qui constituent les piliers essentiels des travaux actuels.

Depuis 2009 ( $c f$. ci-dessus les deux derniers projets), l'équipe de recherche s'est fixée comme but l'édition d'un dictionnaire qui doit rassembler les résultats des recherches effectuées au long des différents projets et dont la parution est prévue pour 2014. Cet ouvrage est conçu à partir d'un double point de vue: d'une part, l'exposé de l'état précis de chaque question (à savoir, les différentes entrées qui ont été fixées); d'autre part, la constitution d'un réseau de déterminations ou d'influences dans les grands domaines (voir plus loin, la proposition d'un classement thématique des recherches).

Il faut signaler également que les thèses de doctorat soutenues en Espagne, concernant l'histoire de l'enseignement du français, abordent toute une série d'aspects majeurs de l'histoire de l'enseignement du FLE, qui vont de l'étude des grammaires et des manuels de français jusqu'aux méthodologies employées ${ }^{6}$, sans oublier la formation et la place des professeurs, ou les lois, règlements et plans d'études qui leur servent de cadre ${ }^{7}$. Des études pionnières ont vu le jour, concernant des figures incontournables telles que Pierre-Nicolas Chantreau ${ }^{8}$ ou des établissements modèles comme l'Institución Libre de Enseñanza (1876-1939) ${ }^{9}$, ainsi que des approches spécialement pointues s'intéressant à la traduction ${ }^{10}$ ou à la lexicographie ${ }^{11}$ où le culturel ${ }^{12}$ représente d'ailleurs un atout majeur. De là peut-être l'attrait pour cette thématique de la part des jeunes chercheurs.

Dans cette présentation d'ensemble, il faut mettre en relief que quatre colloques de la SIHFLES se sont tenus en Espagne : "L"universalité" du français et sa présence dans la Péninsule Ibérique ", en 1995 à Tarragone (Universitat Rovira i Virgili), «Les auteurs classiques français dans l'enseignement du FLE ( $18^{\mathrm{e}}$ et $19^{\mathrm{e}}$ siècles) ", en 1997 à Avila (UNED), «Du maître de langues au professeur de langues (XVIII ${ }^{\mathrm{e}} \mathrm{XIX} \mathrm{X}^{\mathrm{e}}$ siècles): institutionnalisation, professionnalisation et disciplinarisation ", en 2004 (Universidad de Valencia) et «Les rapports entre langues dans les contextes éducatifs en Europe : médiations, circulations, rivalités (XVI - début du XXe siècle)», en 2008 à Grenade (Universidad de Granada).

9 Mais aussi, comme nous l'avons signalé plus haut, de nombreux travaux de toutes sortes (articles de revues, chapitres de livres, monographies) ont été élaborés par de nombreux chercheurs espagnols appartenant à la SIHFLES, et également par d'autres chercheurs, qu'il est intéressant de connaître. Cependant, nous estimons qu'une présentation d'un recueil bibliographique par ordre alphabétique des auteurs n'est qu'une étape nécessaire, mais insuffisante, car elle ne met pas en relief les différents aspects que peut revêtir l'histoire de l'enseignement du français. Nous proposons ainsi une organisation thématique des travaux publiés qui, si l'on veut bien en écarter les défaillances (notamment la proximité inévitable des sujets de recherche, la pluralité thématique de certains travaux, et donc les problèmes de classement), permet de faire un bilan autant que de marquer les lignes de travail à exploiter. Bien sûr, cette proposition devra être confrontée à d'autres, entreprises par les équipes de chercheurs d'autres pays, pour arriver à un classement commun, ce qui constituera un puissant outil de recherche pour les futures études.

10 Nous proposons ainsi de classer les travaux selon le plan suivant, tout en portant une première (et très brève) analyse pour chaque domaine :

11 1. Dimension linguistique et culturelle (les contenus d'enseignement). Ce domaine regroupe une grande partie des travaux (répertoires, analyse des contenus 
d'enseignement, constitution des outils, etc.). Nous ne pouvons pas dire cependant que tout a été fait, loin de là.

\subsection{Les grammaires}

Les études des grammaires pour l'enseignement du FLE abordent pratiquement tous les époques, à partir du XVI ${ }^{\mathrm{e}}$ siècle (Grammaire française de Baltasar de Sotomayor, Alcalá de Henares, 1565) pour se centrer surtout dans ce moment d'exception de l'enseignement du français en Espagne qu'est le XIX ${ }^{e}$ siècle, où le recours à la grammaire bat son plein, sans délaisser pour autant d'autres moments historiques.

\subsection{Le lexique, la lexicologie, la lexicographie}

L'étude du lexique a été l'un des points forts de la bibliographie produite par les chercheurs espagnols. Il faut surtout signaler des approches comparatives concernant la bibliographie franco-espagnole qui devient un atout majeur de cette recherche.

\subsection{La phonétique et l'orthographe}

Les problèmes que ces disciplines posent à l'apprenant hispanophone ont constamment intéressé les spécialistes. Le grand nombre d'études produites dans ce domaine abordent pratiquement tous les aspects qui ont trait à leur apprentissage.

2. Domaine culturel : les aspects culturels / la civilisation / la littérature / les idées / la science / la religion / la morale / les vertus civiques.

Si quelque chose mérite d'être souligné à cet égard, c'est la conclusion partagée par les divers auteurs, à savoir que ces aspects sont étroitement liés à l'enseignement du FLE en Espagne.

3. Dimension sociologique.

Il faut signaler qu'un grand nombre d'articles publiés dans les numéros 18 et 33/34 de Documents, ainsi que certaines thèses signalées supra, ont permis de progresser de façon significative dans cette approche. Il reste cependant beaucoup à faire, et surtout avancer dans la systématisation des travaux, pour avoir des vues d'ensemble sur les évolutions produites dans le profil du maître, le rôle des auteurs, la disciplinarisation du français, l'intervention de l'État, etc.

\subsection{Les acteurs : maîtres et élèves ; auteurs}

3.2. Les institutions / les contextes éducatifs

3.3. Les politiques linguistiques (législation, plan d'études, organisation des enseignements)

4. Dimension didactique et méthodologique (outils, activités, méthodes, théorisations).

À ce sujet, il reste beaucoup à faire, étant donné que ce sont des études particulières (consacrées à un auteur précis, par exemple) qui ont été surtout abordées, sauf pour le rôle de la traduction, comme procédé de travail pendant le XIX ${ }^{\mathrm{e}}$ siècle.

4.1. Les théories sous-jacentes : la langue (linguistique) et l'apprentissage d'une langue (psychologie, psycholinguistique)

4.2. Traduction et enseignement

4.3. Contacts entre langues, plurilinguisme

5. Aspects historiques.

Les aspects historiques, étant donné la perspective même de notre discipline, n'ont pas manqué, abordant pratiquement tous les siècles, depuis la Renaissance jusqu'aux 
époques les plus récentes. Il reste à donner une vue d'ensemble pour comprendre cette histoire dans la longue durée.

Cette bibliographie thématique permet d'embrasser à elle seule la richesse des sujets qui ont mérité l'attention et les efforts des chercheurs espagnols, mais également la variété des approches méthodologiques. En effet, trois courants de recherche s'y trouvent impliqués: une approche plutôt linguistique, une approche plutôt méthodologique, et une approche plutôt historique / culturelle (qui engloberait également la sociologie). Nous sommes conscients que cette délimitation n'est point étanche, et que la linguistique se fait aussi dans son évolution historique, en rapport au changement des idées et des paradigmes. De là toute la difficulté et toute la richesse de notre entreprise, constitutivement interdisciplinaire et plurilingue.

Les travaux réalisés constituent bien sûr une première phase de la recherche. Il faut dire en effet que c'est la pointe d'un iceberg qui a été mise en lumière : il reste à approfondir ce domaine de recherche, autant par des travaux particuliers que par des vues d'ensemble, comme le proposait André Reboullet déjà en 1987 : « la recherche sur l'enseignement du français langue étrangère n'est plus ce qu'elle était: un véhicule sans rétroviseur qui brûle les étapes [...] C'est l'heure des bilans et des rétrospectives » (1987: 208, 56-60).

\section{BIBLIOGRAPHIE}

BRUÑA CUEVAS, Manuel (éd.) (2008a). « La producción lexicográfica con el francés y el español durante los siglos XVI a XIX », Lexicografía bilingüe y plurilingüe del español (siglos XV-XIX) (= Philologia Hispalensis, nํ2). Sevilla : Facultad de Filología de la Universidad de Sevilla, 37-111.

FERNÁNDEZ FRAILE, Ma Eugenia (1995). La enseñanza/aprendizaje del francés en España entre 1767 y 1936 : objetivos, contenidos y procedimientos. Granada : EUG. Thèse doctorale comprenant une importante bibliographie.

FISCHER, Denise, GARCÍA BASCUÑANA, Juan F., JIMÉNEZ, M ${ }^{a}$ Trinidad (2004). Repertorio de gramáticas y manuales de francés para la enseñanza del francés, 1565-1940. Barcelona: PPU.

GONZÁLEZ PALENCIA, Angel (1942). « Notas sobre la enseñanza del Francés a fines del siglo XVIII y principios del XIX », Revista Nacional de Educación (Madrid) 23, año II, 26-34. Réimprimé dans le nº 190, et réédité dans Eruditos y libreros en el siglo XVIII. Estudios histórico-literarios, quinta serie. Madrid : CSIC, Inst. Antonio de Nebrija, 1948, 417-427.

LÓPEZ BARRERA, Joaquín (s.a., début XX). Trabajo de investigación : Apuntes para una historia de la enseñanza de la lengua francesa en España. Cuenca : Celedonio León.

REBOULLET, André (1987). « Pour une histoire de l'enseignement du FLE », Le français dans le monde, 208, 56-60.

SUÁREZ GÓMEZ, Gonzalo (2008). La enseñanza del francés en España hasta 1850. ¿Con qué libros aprendían francés los españoles? de Gonzalo Suárez Gómez. Réédité avec des notes et une étude introductive par J. García Bascuñana \& E. Juan Oliva. Barcelona : PPU. 
SUPIOT, Alberto (1996). « Les manuels de FLE en Espagne entre 1648 et 1815. Approche bibliographique ». Documents pour l'histoire du français langue étrangère ou seconde, 18, 313-338.

SUSO LÓPEZ, Javier, FERNÁNDEZ FRAILE, Mํㅡㄹ Eugenia (2008). Repertorio de manuales para la enseñanza del francés en España. Siglo XX. Granada : Comares.

SUSO LÓPEZ, Javier (2009). « Apuntes para una historia de la enseñanza de la lengua francesa ». In A. Martínez González (éd.). Historia de las ideas lingüísticas. Gramáticos de la España meridional. Francfurt : Peter Lang, 161-170.

YLLERA, Alicia (1998). « Rivalidades lingüísticas franco-españolas en el siglo XVI ». Epos, 14, 383-407.

\section{NOTES}

1. Cet article reprend et met à jour l'étude de Suso López (2009). « Apuntes para una historia de la enseñanza de la lengua francesa ». In Antonio Martínez González (éd.). Historia de las ideas lingüísticas. Gramáticos de la España meridional. Frankfurt : Peter Lang, 161-170.

2. C'est-à-dire titulaire d'une chaire de français dans un lycée.

3. Le mémoire de recherche de J. López Barrera est cité comme source par Suárez Gómez, et mentionné en 1998 par A. Yllera.

4. Il faut aussi faire état des thèses de doctorat de Núria Moreu (1988) et de Quintín Calle (1989), cf. note 7 .

5. Les six projets sont: 1 . Histoire de l'enseignement du français (XVI ${ }^{\mathrm{e}}-\mathrm{XIX}{ }^{\mathrm{e}}$ siècles). PB94-1074 (1995-1998) ; 2. Histoire de l'enseignement du français (XVI'-XIX' siècles). PB97-0410 (1998-2001) ; 3. Contribution à l'histoire de l'enseignement des langues étrangères : présence et enseignement du français en Espagne (1500-1970). BFF 2001-1062 (2001-2004); 4. Grammaires et manuels de français publiés en Espagne: histoire, méthodologie, contenus (1565-2000), BF2001-1062 (2004-2007) ; 5. Élaboration d'un dictionnaire d'histoire de la présence et enseignement du français en Espagne (XVI ${ }^{\mathrm{e}}-\mathrm{XX}^{\mathrm{e}}$ siècles), FFI2008-02389/FILO] (2008-2011); 6. Élaboration d'un dictionnaire d'histoire de la présence et enseignement du français en Espagne. Suite et conclusion, FFI2011-23109 (2011-2014).

6. Francisco Morales (2003). La evolución metodológica de la enseñanza oficial del francés en España: 1936-1970. Universidad de Huelva.

7. Quintín Calle Carabias (1989). La enseñanza oficial de idiomas en España. Por una redefinicion de la formacion teorica del profesorado. Universidad de Málaga; Ma Eugenia Fernández (1995). La enseñanza/aprendizaje del francés en España entre 1767 y 1936: objetivos, contenidos y procedimientos. Universidad de Granada ; Mª Josefa Castellví (1997). Estudio de los métodos de lengua francesa en la primera mitad del siglo XIX como transmisores de una concepción educativa: implícitos didácticos, metodológicos y socioculturales. Universidad de Valencia.

8. Núria Moreu (1988). Pierre-Nicolas Chantreau (1741-1808) et sa grammaire. Universitat de Barcelona.

9. Inmaculada Rius Dalmau (2006). La enseñanza del francés en el marco de la Institución Libre de Enseñanza. Universitat Rovira i Virgili - Tarragona.

10. Esther Juan Oliva (2003). La traducción en los manuales de francés publicados en España durante el siglo XIX, con un apéndice documental en el que se recogen las fichas analíticas de un centenar de manuales. Madrid : UNED.

11. Ana María Carranza Torrejón (2012). El vocabulario de la indumentaria de los siglos XVI a XIX. Estudio contrastivo a partir de las nomenclaturas con el francés y el español. Universidad de Sevilla. 
12. Brigitte Urbano (2003). La enseñanza aprendizaje del francés como lengua extranjera en España durante el franquismo (1938-1970). Universidad de Granada.

\section{AUTEURS}

JUAN FRANCISCO GARCÍA BASCUÑANA

Université Rovira i Virgili, Tarragone

JAVIER SUSO LÓPEZ

Université de Grenade 